\title{
Design and Analyze the Optimum Operating Point between Magnetic Flux Density and Vibration Noise of Transformer Cores
}

\author{
Lihua Zhu*, Xin Zhang, Qingxin Yang and Xian Zhang
}

Municipal Key Laboratory of Advanced Technology of Electrical Engineering and Energy, Tianjin Polytechnic University; Tianjin, 300387, China

\begin{abstract}
Base on the magnetization and magnetostriction measurement, this paper proposed a generalized 3D finite element numerical model, which can be used to analyze electromagnetic vibration of laminated cores including electromagnetism and magnetostriction effects. And then the acoustic field around the transformer was analyzed and tested, the results showed the model and analysis method were feasible. The work is helpful to estimate vibration noise level and then optimized design, choose suitable silicon steel and size in the design stage, which can reduce product development cycle and test cost.
\end{abstract}

Keywords: Operating point, magnetic flux density, vibration noise, acoustic field, magnetostriction.

\section{INTRODUCTION}

Energy saving and noise reduction must be the development trend of power transformers. Improving the magnetic flux density in cores can reduce the size of transformers, which will reduce the cost for the equipment and materials such as silicon-iron, copper, carbon steel, and insulation material. However, the vibration noise of cores will boost as the magnetic flux density increases. So, it is the part that needs optimization due the high cost and low vibration noise related with final product.

Electromagnetic vibration noise of power transformers under no load, especially dry transformers, mainly caused by silicon steel magnetostriction (MS) under the nominal operating flux density $1.5 \mathrm{~T}-1.8 \mathrm{~T}[1,2]$. Several papers have analyzed the vibration of cores due to magnetostrictive effect in 2D, some based the Principle of virtual work [3], and others based the equations of elasticity method in a similar way as thermal stresses [4-6]. The aim of this paper was to investigate a feasible way to determine the operating point of transformer cores within the vibration noise limit set by the government.

In this paper, the magnetic and MS characteristics of the oriented silicon steel both in the rolling direction (RD) and transverse direction (TD) were firstly measured which is necessary to the vibration analysis of transformer cores. Because the vibration of laminated cores due to MS is not only in the plane of the sheets, but also out-of-plane [7, 8], so a 3D numerical model including MS effects and magnetic anisotropy was developed, which was used to analyze the magnetic flux density and deformation of laminated cores. The acoustic field was also analyzed based on the total sound power emitted by the cores and windings. Finally, the noise level was investigated through analysis and measured results, which confirmed the reliability of the presented model and analysis method and provided a way to determine the optimum operating point of transformer cores between the magnetic flux density and vibration noise level in the design progress.

\section{METHODES OF ANALYSIS}

\subsection{Magnetization and Magnetostriction Measurement}

In order to measure the magnetization curves of silicon steel sheet of the transformer prototype, standard samples with $30 \mathrm{~mm} * 300 \mathrm{~mm}$ were respectively cut $0^{\circ}$ and $90^{\circ}$ intervals from $\mathrm{RD}$ of the oriented steel. The strips, cut at each angle, stacked in $25 \mathrm{~cm}$ Epstein Frame, then the magnetic anisotropy were tested according to GB/T 3655-2008. The hysteresis loops of one type grain oriented steel in RD from measurement were shown in Fig. (1a). Magnetostriction is the intrinsic characteristics of laminated core and is dependent on the magnetic induction. To analyze the vibration of the transformer cores including MS effects, the MS strains both in RD and TD are obtained with a $100 \mathrm{~mm} * 500 \mathrm{~mm}$ sample piece by a heterodyne laser vibrometer, which meet the international standard IEC/TR62581 [9]. The alternating magnetization MS butterfly curves in RD under different induction values $B_{\mathrm{RD}}$ as demonstrated by Fig. (1b).

To simplified calculation program, the hysteresis and butterfly characters under alternating magnetic were ignored in this paper. Considering magnetic anisotropy of the grain oriented steel, magnetization and MS curves in RD and TD, used to analyze the magnetic field and vibration due to MS of transformer cores, were measured and shown in Fig. (2). 


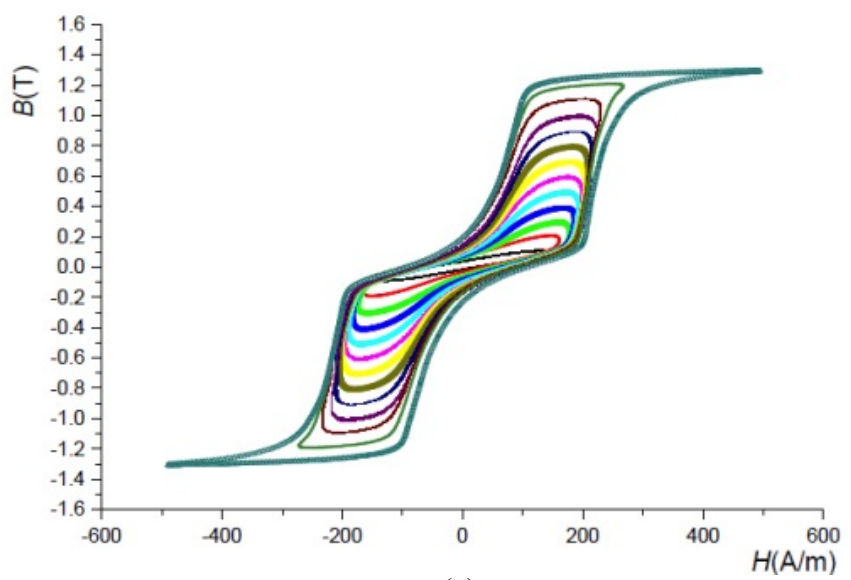

(a)

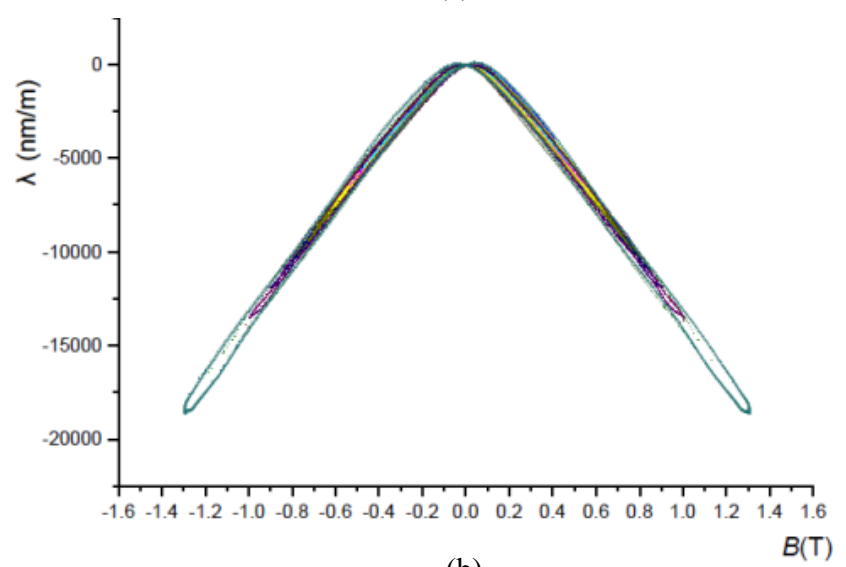

(b)

$B(T)$

Fig. (1). AC magnetic characteristics curves of one type grain oriented steel in RD with $50 \mathrm{~Hz}$. (a) Hysteresis loops. (b) Magnetostricon butterfly curves

\subsection{Functional Energy of Cores}

Based on previous researches [8], the MS behavior of silicon steel materials follows piezomagnetic laws which is rewritten as:

$\begin{cases}\varepsilon_{i}=s_{i j}^{H} \sigma_{j}+d_{n i} H_{n} & i, j=1, \ldots 6 \\ B_{m}=d_{m j} \sigma_{j}+\mu_{m n}^{\sigma} H_{n} & m, n=1, \ldots 3\end{cases}$

where $s^{H}, \boldsymbol{d}, \mu^{\sigma}$ are the tensors matrix of constant- $H$ compliance, MS coefficients and constant- $\sigma$ permeability matrix, $\varepsilon$ and $\sigma$, the tensors of strain and stress, $\boldsymbol{B}$ and $\boldsymbol{H}$, the vectors of magnetic flux density and magnetic field, respectively.

The total energy functional of the transformer cores include mechanical energy, magnetic energy and magnetmechanical coupling energy. It can be expressed as follows:

$$
\begin{aligned}
I=\int_{\Omega_{2}}\left(\frac{1}{2} \sigma^{T} \boldsymbol{s}^{H} \sigma\right) d V+\int_{\Omega_{2}}\left(\sigma^{T} \boldsymbol{d} \boldsymbol{H}\right) d V+ \\
\int_{\Omega_{1}}\left(\frac{1}{2} \boldsymbol{H}^{T} \boldsymbol{\mu}^{\sigma} \boldsymbol{H}\right) d V-\int_{\Omega_{1}} \boldsymbol{J} \cdot \boldsymbol{A} d V- \\
\int_{\Gamma_{1}} \boldsymbol{f}_{\Gamma} \cdot \boldsymbol{u} d V-\int_{\Omega_{2}} \boldsymbol{f}_{V} \cdot \boldsymbol{u} d V
\end{aligned}
$$

where $\boldsymbol{A}$ is the magnetic vector potential, and $\mathbf{B}=\nabla \times \mathbf{A} . \boldsymbol{U}$ is the mechanical displacement. $f_{V}$ and $f_{\Gamma}$ are the external volume force and boundary surface force of the transformer cores. $\Omega_{1}$ and $\Omega_{2}$ are the analysis domain of magnetic and mechanical.

\subsection{Anisotropy Analysis}

Magnetic permeability $v=\left(v_{x} v_{y} v_{z}\right)^{\mathrm{T}}$, in coordinate system $v_{x}, v_{y}$ were obtained by interpolation from measured magnetization curves of $B_{x} H_{x}$ and $B_{y} H_{y}$, which is $B_{R D} H_{R D}$ or $B_{T D} H_{T D}$ determined the core position in the coordinate system. $v_{z}=v_{0}$ in lamination thickness direction for the silicon steel sheet were coated with insulating layer.

Based on Eq. (1), the strain of transformer core caused by magnetostriction can be obtained as follows:

$$
\boldsymbol{\varepsilon}^{\prime}=\boldsymbol{d} \cdot \boldsymbol{H}=\left(\begin{array}{llllll}
d_{11} & d_{12} & d_{13} & d_{14} & d_{15} & d_{16} \\
d_{21} & d_{22} & d_{23} & d_{24} & d_{25} & d_{26} \\
d_{31} & d_{32} & d_{33} & d_{34} & d_{35} & d_{36}
\end{array}\right)^{\mathrm{T}}\left\{\begin{array}{c}
H_{x} \\
H_{y} \\
H_{z}
\end{array}\right\}
$$

MS coefficient matrix $d$, in which $d_{11}, d_{22}$ can be obtained from measured MS characteristic curves $\lambda_{x}\left(B_{x}\right)$ and $\lambda_{y}\left(B_{y}\right)$. If 


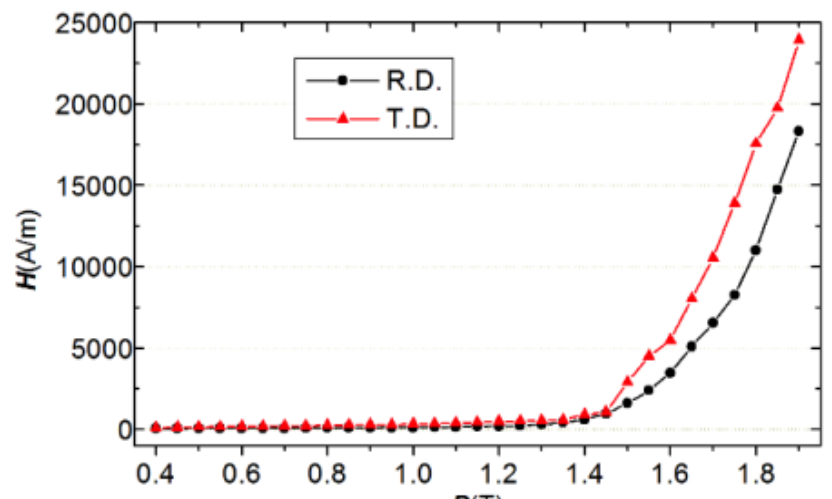

(a) $B(T)$

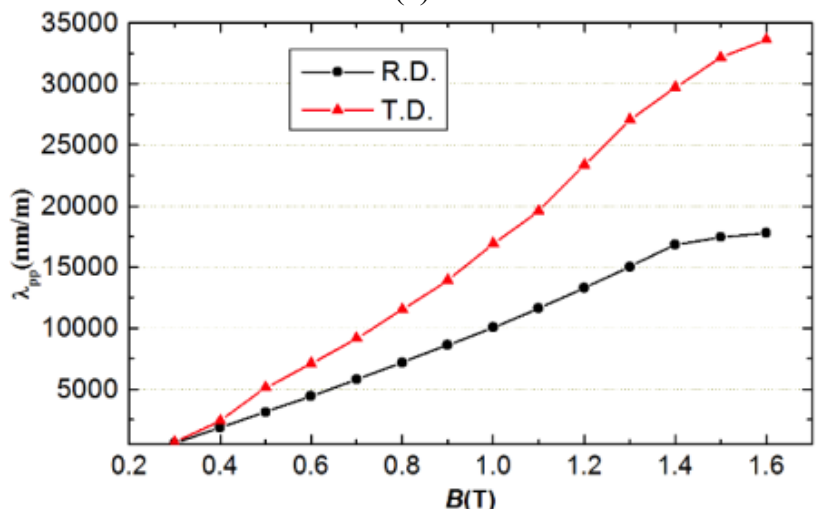

(b)

Fig. (2). The magnetization curves and peak-to-peak MS values in RD and TD of the type grain oriented steel. (a) Magnetization curves. (b) Peak-to-peak MS values.

shearing strains of the steel lamination is neglected, there is $d_{i j}=0 \quad(i=4,5,6, j=1,2,3)$. The MS coefficient in the normal direction is assumed as $d_{33}=\left(d_{11}+d_{22}\right) / 2$. Using the Hooker's law, we can get $d_{21}=d_{31}=-\alpha d_{11}, d_{12}=d_{32}=-\alpha d_{22}, d_{13}=d_{23}=-\alpha d_{33}$, where $\alpha$ is the Poisson ratio.

So, the magneto-mechanical coupling energy of transformer cores is given as:

$$
\begin{aligned}
& \int_{\Omega_{2}} \sigma^{\mathrm{T}} \boldsymbol{d} \boldsymbol{H} \mathrm{d} V= \\
& E^{\alpha} \int_{\Omega_{2}}\left(\begin{array}{c}
(1-\alpha) \varepsilon_{x}+\alpha \varepsilon_{y}+\alpha \varepsilon_{z} \\
\alpha \varepsilon_{x}+(1-\alpha) \varepsilon_{y}+\alpha \varepsilon_{z} \\
\alpha \varepsilon_{x}+\alpha \varepsilon_{y}+(1-\alpha) \varepsilon_{z} \\
(1-2 \alpha) \gamma_{x y} / 2 \\
(1-2 \alpha) \gamma_{y z} / 2 \\
(1-2 \alpha) \gamma_{z x} / 2
\end{array}\right)\left(\begin{array}{ccc}
1 & -\alpha & -\alpha \\
-\alpha & 1 & -\alpha \\
-\alpha & -\alpha & 1 \\
0 & 0 & 0 \\
0 & 0 & 0 \\
0 & 0 & 0
\end{array}\right)\left(\begin{array}{c}
d_{11} H_{x} \\
d_{22} H_{y} \\
d_{33} H_{z}
\end{array}\right)^{\mathrm{T}} \mathrm{d} V(4) \\
& =E \int_{\Omega_{2}}\left(d_{11} v_{x} B_{x} \varepsilon_{x}+d_{22} v_{y} B_{y} \varepsilon+d_{33} v_{z} B_{z} \varepsilon_{z}\right) \mathrm{d} x \mathrm{~d} y \mathrm{~d} z
\end{aligned}
$$

where, $E^{\alpha}=\frac{E(1-\alpha)}{(1+\alpha)(1-2 \alpha)}$, and $E$ is the Young's modulus of the cores.
After element discretization of functional $I$ and element assembly, then matrix equation of the magneto-elastic system is given by

$$
\left(\begin{array}{cc}
M & D \\
C & K
\end{array}\right)\left(\begin{array}{l}
A \\
u
\end{array}\right)=\left(\begin{array}{c}
J \\
f_{v}+f_{*}
\end{array}\right)
$$

where $\boldsymbol{M}$ is the electromagnetic matrix, $\boldsymbol{K}$, the mechanical stiffness matrix, $\boldsymbol{C}, \boldsymbol{D}$, the coupling interactions between the magnetic field and mechanical deformation, and $\boldsymbol{C}=\boldsymbol{D}^{T}$. Magnetic- mechanical including MS is coupled by the $C$ matrix.

\subsection{Acoustical Analysis}

Based on the vibration calculation, according to classical theoretical [10], the sound power of the test position radiated by cores can be expressed as:

$$
\begin{gathered}
W_{i}=\rho c \kappa_{j} \oint_{S} v_{n}^{2} d S_{c}=\rho_{0} c_{0} \kappa_{j} \sum_{j} v_{n, k}^{2} S_{c, k} \cos \theta_{i} \\
=\rho_{0} c_{0} \kappa_{j} \sum_{j}\left(\frac{\partial u_{n, k}}{\partial t}\right)^{2} S_{c, k} \cos \theta_{i}
\end{gathered}
$$


Table 1. Main parameters of the transformer.

\begin{tabular}{|c|c|}
\hline Parameter Names & Values \\
\hline \hline Rated capacity & $10(\mathrm{kVA})$ \\
\hline rated voltage & $1000 / 380(\mathrm{~V})$ \\
\hline rated current & $5.77 / 15.2(\mathrm{~A})$ \\
\hline connection symbol & Yy0 \\
\hline no-load loss & $72(\mathrm{~W})$ \\
\hline no-load current & $2.0(\%)$ \\
\hline
\end{tabular}

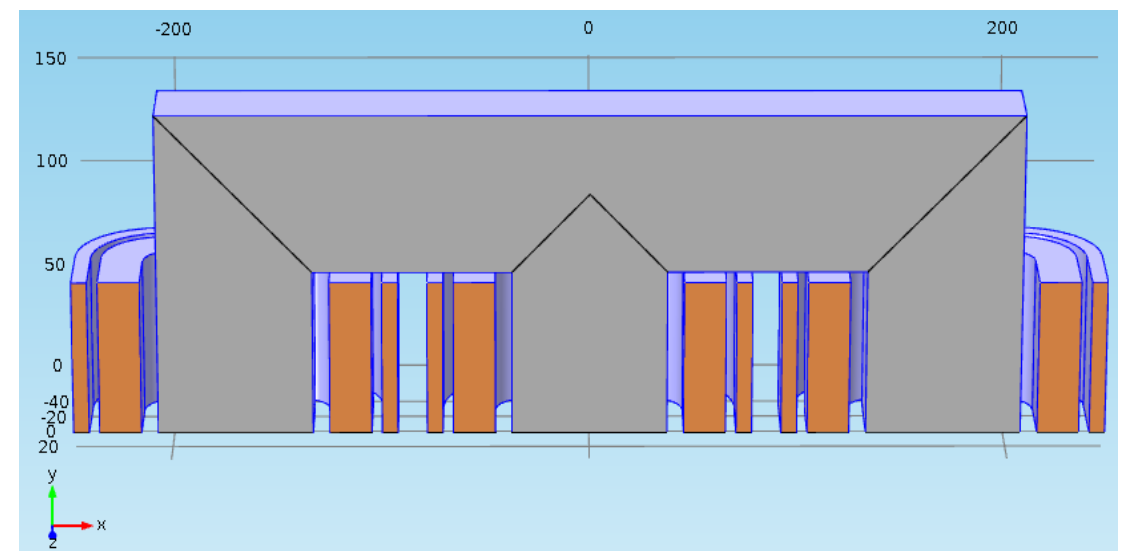

Fig. (3). Analysis model of the transformer.

where $\rho c$ is the characteristic impedance of the noise transmission medium, $k_{j}$ the radiation coefficient of the $j$ core surface, $\cos \theta_{i}$ is the angle between the test point and the surface unit $d S_{c i}$ of transformer cores.

Then we can get the sound pressure level $L_{p}$ of the free sound field around the core:

$L_{p i}=10 \lg \left(W_{i} / W_{0}\right)-\lg R_{i}-D$

$W_{i}$ is the sound power calculated by Eq. (6), $W_{0}=10^{-12} \mathrm{~W}$ is the reference's value. $R_{i}$ the distance from the measured point to the core.

\section{RESULTS AND DISCUSSION}

The numerical model and vibration tests were implemented on a dry power transformer and the main parameter of the transformer is shown in Table (1).

The magnetic-mechanical strong coupling was calculated by finite element of $1 / 4$ according to the symmetry and the analysis model of the dry-type power transformer in the rectangular coordinate as shown in Fig. (3). The magnetic field and deformation of the transformer core can be obtained from numerical analysis. The magnetic flux distribution and deformation in different time is displayed in Fig. (4). It is evident that the deformation is bigger where with larger magnetic flux, which is consistent with the theoretical and Eq. (1).

According to China's national standard GB7328-87, the analysis and test points of the acoustic field are shown in Fig. (5). The no-load noise was measured by AWA6270 analyzer as shown in Fig. (6). Based the magneto-elastic analysis, the sound level around the dry transformer were calculated and the result in a moment as Fig. (7) shown, which including the windings in order to close the actual working and measuring condition.

Sound pressure level results of the points, shown in Fig.5, from analysis and measured were listed in Table 2 when the transformer cores were inspired by rated voltage. From the table we can see the maximal error between analysis and measurement is $3.37 \%$, which in the range of $5 \%$ allowed by engineering. So, it's feasible to estimate the noise level emitted by cores and winding based the proposed model and method in the design stage. The optimum operating point of transformer cores can be achieved through changing flux density and material of cores considering product cost and noise limit. 


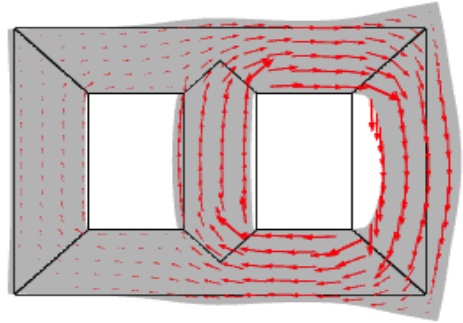

(a) $2 \mathrm{~ms}$

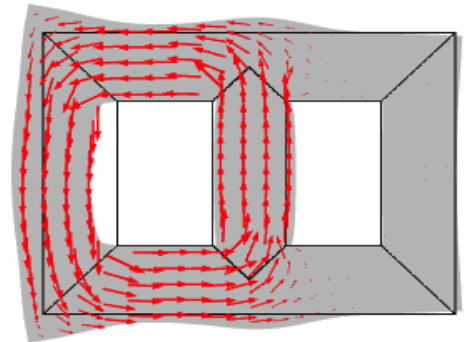

(c) $14 \mathrm{~ms}$

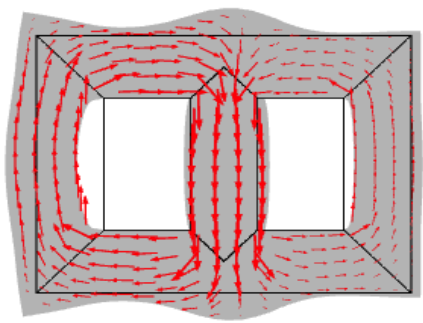

(b) $6 \mathrm{~ms}$

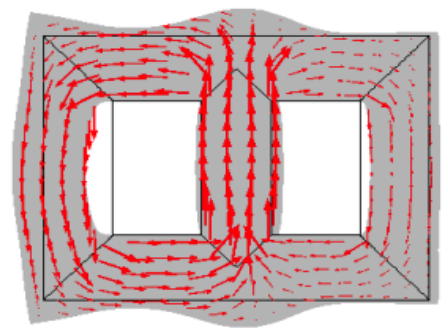

(d) $16 \mathrm{~ms}$

Fig. (4). Section view of magnetic flux and deformation distribution of the transformer core.

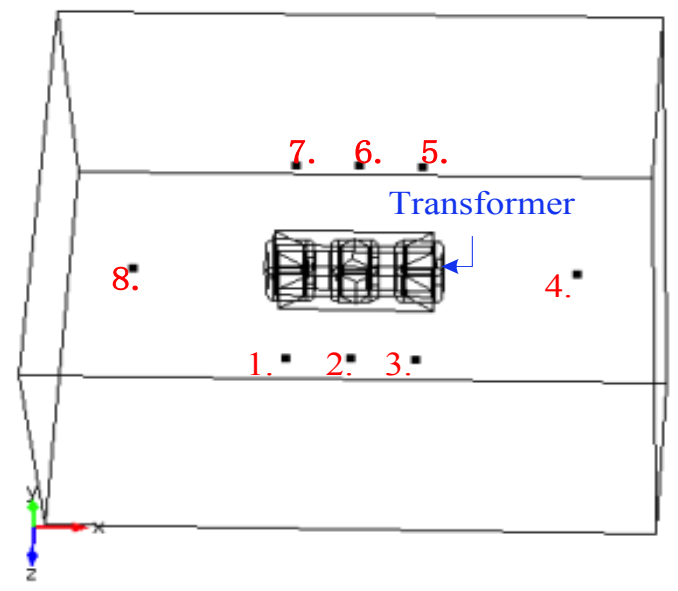

Fig. (5). The acoustic field and measuring points.

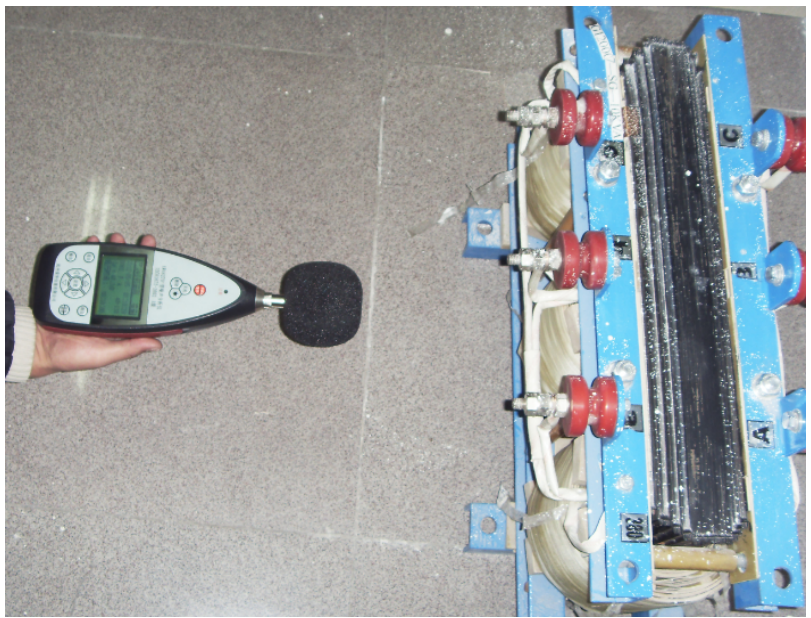

Fig. (6). Sound pressure level measurement. 


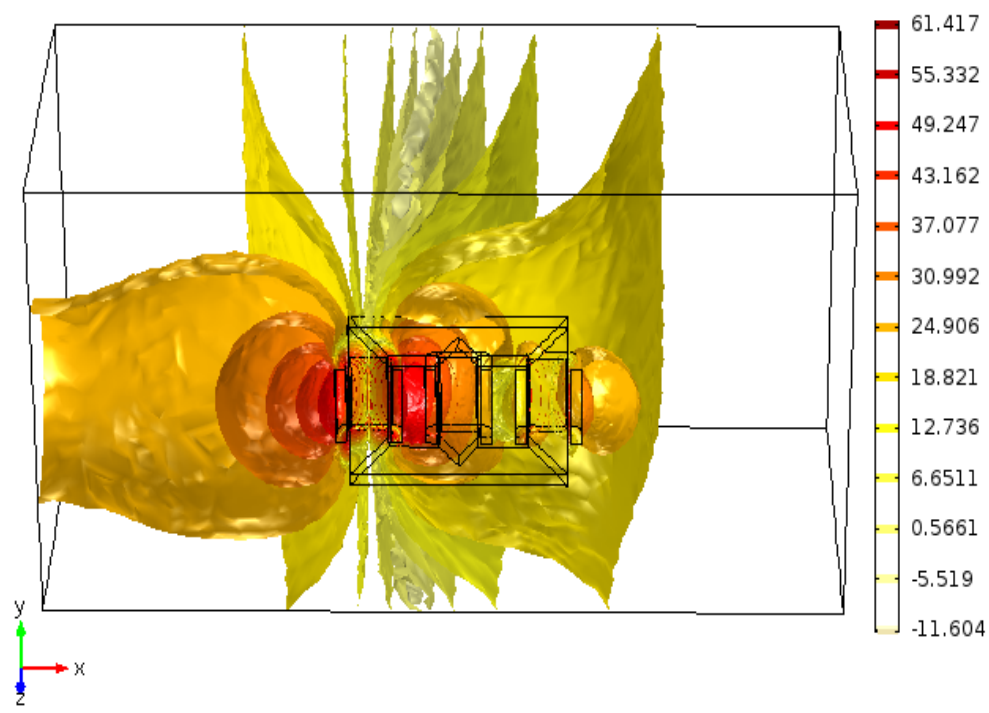

Fig. (7). Sound pressure level contour surface from analysis in a moment.

Table 2. The result of sound level from analysis and measurement.

\begin{tabular}{|c|c|c|c|}
\hline Points & $\begin{array}{c}\text { Analysis } \\
\text { (dB) }\end{array}$ & $\begin{array}{c}\text { Measurement } \\
\text { (dB) }\end{array}$ & $\begin{array}{c}\text { Errors } \\
(\%)\end{array}$ \\
\hline 2 & 30.3 & 29.9 & +1.34 \\
\hline 3 & 27.6 & 28.1 & -1.78 \\
\hline 5 & 29.6 & 30.3 & -2.31 \\
\hline 6 & 30.5 & 31.2 & -2.24 \\
\hline 7 & 30.1 & 30.8 & -2.27 \\
\hline
\end{tabular}

\section{CONCLUSION}

A numerical model and analysis method for predicting vibration and noise of transformer products is proposed in this paper, which is subject to solve the contradiction between cost and noise limits. Applying the model, the vibration noise of a transformer was analyzed and measured and the results confirmed the correctness of the model and feasible of the method. In a word, the work is helpful to design the optimum operating point of transformer cores, shorten new product development cycle and reduce costs.

\section{CONFLICT OF INTEREST}

There is no conflict of interest between financial contributions to the paper work.

\section{ACKNOWLEDGEMENTS}

The research work is supported by National Natural Science Foundation of China $(51237005,51177038)$ and Natural Science Foundation of Tianjin (12JCDJ286000).

\section{REFERENCES}

[1] R. Zimbelman, "A Contribution to the Problem of CementAggregate Bond", Cement and Concrete Research, vol. 15, pp. 801-8, June 1985.

[2] L. Lahn, C.Wang, A. Allwardt, T. Belgrand, and J. Blaszkowski, "Improved transformer noise behaviour by optimized laser domain refinement at thyssenkrupp electrical steel," IEEE Transactions on Magnetics, vol. 48, no. 4, pp. 1453-6, Apr. 2012.

[3] B. Weiser, H. Pfützner, J.Anger, "Relevance of magnetostriction and forces for the generation of audible noise of transformer cores", IEEE Transactions on Magnetics, vol.36, no.5, pp. 375977, Sept. 2000 
[4] Mohammed O A, Calvert T E, Mcconnell R, "Coupled magnetoelastic finite element formulation including anisotropic reluctivity tensor and magnetostriction effects for machinery applications", IEEE Transactions on Magnetics, vol.37, no.5, pp. 3388-3392, 2001.

[5] Witod Kubiak, Pawel Witczak. "Vibration analysis of small power transformer", The International Journal for Computation and Mathematics in Electrical and Electronic Engineering, vol.29, no.4, pp: 1116-24, 2010.

[6] Lieven Vandevelde, Jan A A Melkebeek. "Magnetic forces and magnetostriction in electrical machines and transformer cores", IEEE Transactions on Magnetics, vol.29, no.2, pp: 1618-21, 2002.

[7] G. Yanhui, M. Kazuhiro, I. Yoshiyuki, et al. "Vibration analysis of a reactor driven by an inverter power supply consider electromag- netism and magnetostriction", IEEE Transactions on Magnetics, vol.45, no.10, pp: 4789-4792, 2009.

[8] W. Kubiak and P. Witczak, "Vibration analysis of small power transformer," COMPEL, vol. 29, no. 4, pp. 1116-1124, 2010.

[9] Lihua Zhu, Qingxin Yang, Rongge Yan, et al. "Numerical computation for a new way to reduce vibration and noise due to magnetostriction and magnetic forces of transformer cores", Journal of Applied Physics, vol.113, no.17, pp: 17A333,May, 2013.

[10] IEC/TR 62581[S], Electrical Steel-Methods of measurement of the magnetostriction characteristics by means of single sheet and Epstein test specimens, 2010 .

[11] Taibao Li, Computational acoustics: Sound field equation and calculation method, Beijing: Science Press, 2005.

Received: October 16, 2014

Revised: December 23, 2014

Accepted: December 31, 2014

(C) Zhu et al.; Licensee Bentham Open.

This is an open access article licensed under the terms of the Creative Commons Attribution Non-Commercial License (http://creativecommons.org/licenses/by$\mathrm{nc} / 3.0 /$ ) which permits unrestricted, non-commercial use, distribution and reproduction in any medium, provided the work is properly cited. 